\title{
DUALITY FOR REPRESENTATIONS OF A HECKE ALGEBRA
}

\author{
SHIN-ICHI KATO
}

(Communicated by Jonathan M. Rosenberg)

\begin{abstract}
We describe a duality operator for representations of the Hecke algebra of a Weyl group or of an affine Weyl group in terms of a certain involution on this algebra.
\end{abstract}

For representations of finite Chevalley groups, several authors studied the duality operator, an endomorphism on the Grothendieck group of these representations, defined to be an alternating sum of some combinations of induction and restriction (see $[\mathrm{A}, \mathrm{C}, \mathrm{Kw}, \mathrm{DL}])$. In this note, we define a duality operator for representations of the Hecke algebra of a Weyl group, or of an affine Weyl group, which is a counterpart of the above one, and describe it in terms of a certain involution on this algebra.

\section{THE CASE OF WeYL GROUPS}

Let $(W, S)$ be a pair consisting of a Weyl group $W$ and a set of simple reflections $S$, or, more generally, a finite Coxeter system. Let $K$ be an arbitrary field. Choose $q_{s} \in K^{\times}$for each $s \in S$ so that $q_{s}=q_{s^{\prime}}$ if $s$ and $s^{\prime}$ are conjugate under $W$. For these $\left(q_{s}\right)_{s \in S}$, we define the Hecke algebra $H=H(W, q)$ over $K$ as follows: $H$ is a $K$-algebra with basis $T_{w}(w \in W)$ which satisfies the relations

$$
\begin{gathered}
\left(T_{s}+1\right)\left(T_{s}-q_{s}\right)=0 \quad(s \in S), \\
T_{w} \cdot T_{w^{\prime}}=T_{w w^{\prime}} \quad \text { if } l(w)+l\left(w^{\prime}\right)=l\left(w w^{\prime}\right) .
\end{gathered}
$$

Here $l: W \rightarrow \mathbb{Z}_{\geq 0}$ is the length function with respect to $S$. For simplicity we assume $q_{s}=q$ for all $s \in S$ in the following, although our argument in $\S \S 1$ and 2 below is equally valid in the general case. For $I \subset S$, let $W_{I}$ be the subgroup of $W$ generated by $I$ and let $H_{I}$ be the corresponding subalgebra of $H$ generated by $T_{w} \quad\left(w \in W_{I}\right)$. Then for any $H_{I}$-module $N$, we can define the induced module $\operatorname{Ind}_{I} N$ by $\operatorname{Ind}_{I} N=H \otimes_{H_{I}} N$. Also we denote by $\operatorname{Res}_{I} M$ for an $H$-module $M$ the $H_{I}$-module obtained by restriction to $H_{I}$. Let $\mathscr{R}$ be the Grothendieck group of finite-dimensional $H$-modules over $K$, and let [M] be the element in $\mathscr{R}$ corresponding to an $H$-module $M$. Now we define the duality operator $D$ on $\mathscr{R}$ by

$$
D[M]=\sum_{I \subset S}(-1)^{|I|}\left[\operatorname{Ind}_{I}\left(\operatorname{Res}_{I} M\right)\right] .
$$

Received by the editors July 5, 1990 .

1991 Mathematics Subject Classification. Primary 22E50; Secondary 20G05. 
When $q=$ prime power and $\operatorname{char}(K)=0$, this operator corresponds to the special case (where representations are generated by the Borel subgroup fixed vectors) of the previously studied operator in [A, C, Kw, DL]. Put $T_{w}^{*}=$ $(-q)^{l(w)} T_{w^{-1}}^{-1}$. This ${ }^{*}$ defines an involutive automorphism of $H$. For an $H-$ module $M$, denote by $M^{*}$ the $H$-module obtained by twisting the action of $H$ by this involution ${ }^{*}$.

Theorem 1. We have $D[M]=\left[M^{*}\right]$ for any $H$-module $M$.

Remark. This theorem is already known for $q=1$ [Sl], $q=$ prime power, and $\operatorname{char}(K)=0$ (where $W$ is a Weyl group) [C, Theorem 1.7], or, more generally, when both $H$ and $K[W]$ are semisimple (Gyoja, unpublished).

In order to prove Theorem 1, we note first that

$\operatorname{Ind}_{I}\left(\operatorname{Res}_{I} M\right)=H \otimes_{K} M /\left\langle h T_{s} \otimes m-h \otimes \pi\left(T_{s}\right) m ; h \in H, s \in I, m \in M\right\rangle$.

Here $\pi$ is the action of $H$ on $M$, and $H$ acts on $H \otimes_{K} M$ by (left multiplication $) \otimes 1$. We define $\tau_{s} \in \operatorname{End}_{K}\left(H \otimes_{K} M\right)$ for $s \in S$ by

$$
\tau_{s}(h \otimes m)=h T_{s} \otimes \pi\left(T_{s}\right)^{-1} m-h \otimes m
$$

and put $L_{s}=\operatorname{Im} \tau_{s}$. Hence we have

$$
\operatorname{Ind}_{I}\left(\operatorname{Res}_{I} M\right)=H \otimes_{K} M / \sum_{s \in I} L_{s} .
$$

Let

$$
\pi_{I}^{I^{\prime}}: H \otimes_{K} M / \sum_{s \in I} L_{s} \rightarrow H \otimes_{K} M / \sum_{s \in I^{\prime}} L_{s}
$$

be the natural projection for $I \subset I^{\prime}$, which is an $H$-homomorphism. Also, to describe the orientation, we let

$$
\varepsilon_{I}^{I^{\prime}}: \bigwedge^{|I|}\left(K^{I}\right) \rightarrow \bigwedge^{\left|I^{\prime}\right|}\left(K^{I^{\prime}}\right) \quad\left(I \subset I^{\prime} \subset S \text { with }\left|I^{\prime}\right|=|I|+1\right)
$$

be the natural isomorphism given by $v \rightarrow v \wedge s \quad\left(s \in I^{\prime} \backslash I\right)$. Here we denote by $K^{X}$ the free $K$-module with basis $X$ for any set $X$ (and put $\wedge^{|I|}\left(K^{I}\right)=K$ for $I=\varnothing)$. Then we can define the following complex of $H$-modules (cf. [DL, part I]):

$$
0 \rightarrow C_{0} \stackrel{d_{0}}{\rightarrow} C_{1} \stackrel{d_{1}}{\rightarrow} \ldots \stackrel{d_{i-1}}{\rightarrow} C_{i} \stackrel{d_{i}}{\rightarrow} \cdots \rightarrow C_{|S|} \rightarrow 0,
$$

where $C_{i}=\bigoplus_{|I|=i}\left(H \otimes_{K} M / \sum_{s \in I} L_{s}\right) \otimes \bigwedge^{i}\left(K^{I}\right)$ and the map $d_{i}$ has the components $\pi_{I}^{I^{\prime}} \otimes \varepsilon_{I}^{I^{\prime}} \quad\left(|I|=i,\left|I^{\prime}\right|=i+1\right.$, and $\left.I \subset I^{\prime}\right)$. Now we shall show that

(\#) this complex has a nonzero cohomology only at the degree 0 .

Let us identify $M^{W}=K^{W} \otimes_{K} M$ with $H \otimes_{K} M$ by the linear map

$$
\varphi: M^{W} \rightarrow H \otimes_{K} M \quad\left(\varphi\left(\left(m_{w}\right)_{w \in W}\right)=\sum_{w \in W} T_{w} \otimes \pi\left(T_{w}\right)^{-1} m_{w}\right)
$$

and forget about the $H$-module structure on $K^{W} \otimes_{K} M$. 
Lemma 1. For $s \in S$, put

$$
K_{s}^{W}=\left\{\left(x_{w}\right)_{w \in W} \in K^{W} ; x_{w s}=-x_{w}(w \in W)\right\} .
$$

Then we have $\varphi^{-1}\left(L_{s}\right)=K_{s}^{W} \otimes_{K} M$.

Proof. Let $y$ be an element of $W$ with $l(y s)>l(y)$. Then for $m_{y}, m_{y s} \in M$, we have

$$
\begin{aligned}
\tau_{s}\left(T_{y}\right. & \left.\otimes \pi\left(T_{y}\right)^{-1} m_{y}+T_{y s} \otimes \pi\left(T_{y s}\right)^{-1} m_{y s}\right) \\
& =-T_{y} \otimes \pi\left(T_{y}\right)^{-1} m^{\prime}+T_{y s} \otimes \pi\left(T_{y s}\right)^{-1} m^{\prime},
\end{aligned}
$$

where

$$
m^{\prime}=m_{y}+q \pi\left(T_{y} \cdot T_{s}^{-2} \cdot T_{y}^{-1}\right) m_{y s} .
$$

This shows that $\varphi^{-1}\left(L_{s}\right) \subset K_{s}^{W} \otimes_{K} M$. Moreover, if we put $m_{y s}=0$ in the above, we get $\varphi^{-1}\left(L_{s}\right) \supset K_{s}^{W} \otimes_{K} M$. Thus we have Lemma 1 .

By this lemma we see that $\varphi$ in (1.3) induces a $K$-isomorphism

$$
\left(K^{W} / \sum_{s \in I} K_{s}^{W}\right) \otimes_{K} M \simeq H \otimes_{K} M / \sum_{s \in I} L_{s} .
$$

Hence we have only to show that the complex

$$
\begin{aligned}
0 & \rightarrow \cdots \rightarrow \bigoplus_{|I|=i}\left(K^{W} / \sum_{s \in I} K_{s}^{W}\right) \otimes \bigwedge^{i}\left(K^{I}\right) \\
& \rightarrow \bigoplus_{|I|=i+1}\left(K^{W} / \sum_{s \in I} K_{s}^{W}\right) \otimes \bigwedge^{i+1}\left(K^{I}\right) \rightarrow \cdots \rightarrow 0
\end{aligned}
$$

defined similarly as (1.2) satisfies (\#). Let $K\left[W / W_{I}\right]$ be the free $K$-module with basis consisting of cosets $w W_{I}$. Since we can identify canonically $K^{W} / \sum_{s \in I} K_{s}^{W}$ with $K\left[W / W_{I}\right]$ by the isomorphism induced by the surjection $K^{W} \rightarrow K\left[W / W_{I}\right] \quad\left(\left(x_{w}\right)_{w \in W} \mapsto \sum_{w \in W} x_{w} \cdot w W_{I}\right)$, we see that the complex (1.4) is identical to the one in [SI] (see also [DL, p. 287]) which arises from a simplicial decomposition of the $(|S|-1)$-dimensional sphere. Thus the condition (\#) holds for (1.4), hence for (1.2).

Proof of Theorem 1. Since the 0th cohomology module of (1.2) is $\operatorname{Ker} d_{0}=$ $\bigcap_{s \in S} L_{s}$, we have

$$
D[M]=\sum_{i}(-1)^{i}\left[C_{i}\right]=\left[\bigcap_{s \in S} L_{s}\right] .
$$

Put $\chi=\sum_{w \in W}(-1)^{l(w)} T_{w} \otimes T_{w}^{-1} \in H \otimes_{K} H$. Then Lemma 1 shows that $\bigcap_{s \in S} L_{s}=(\mathrm{id} \otimes \pi)(\chi)(1 \otimes M)$. Namely, all the elements of $\bigcap_{s \in S} L_{s}$ can be written uniquely as $\sum_{w \in W}(-1)^{l(w)} T_{w} \otimes \pi\left(T_{w}\right)^{-1} m$ for some $m \in M$. But we can see easily (cf. Lemma 2 below) that

$$
\left(T_{s} \otimes 1\right) \chi=\chi\left(1 \otimes\left(-q T_{s}^{-1}\right)\right) \quad(s \in S)
$$

in $H \otimes_{K} H$. Thus $\chi$ intertwines the natural $H$-action and the twisted $H$ action under ${ }^{*}$, which implies that the $H$-module $\bigcap_{s \in S} L_{s}$ is isomorphic to $M^{*}$. This completes the proof of Theorem 1 . 


\section{The CASE OF AFFINE WeYl gRoups}

Let $\left(L, R, L^{\vee}, R^{\vee}\right)$ be the root data (see [Sp]) of a complex reductive group $G$ with respect to a maximal torus $T$; that is, $L=\operatorname{Hom}\left(T, \mathbb{C}^{\times}\right)$(the character group of $T$ ), $L^{\vee}=\operatorname{Hom}\left(\mathbb{C}^{\times}, T\right)$ (the group of 1-parameter subgroups of $T$ ), $R$ is the root system of $(G, T)$, and $R^{\vee}$ is the coroot system. We fix an order on $R$ and denote by $R^{+}$the set of positive roots. Put $W=$ the Weyl group of $(G, T)$ and $S=$ the set of simple reflections. Then $W$ acts on $L$. Let $\widetilde{W}$ be the (generalized) affine Weyl group of $(G, T)$ defined to be the semidirect product of $W$ by $L$. This $\widetilde{W}$ contains $W_{a}=W \ltimes Q \quad(Q=\mathbb{Z} R$ is the root lattice) as a normal subgroup. We assume hereafter that $R$ is irreducible for simplicity. Let $\alpha_{0}^{\vee}$ be the maximal coroot and put $s_{0}=s_{\alpha_{0}} t_{-\alpha_{0}} \in \widetilde{W}$. Here we denote by $s_{\alpha}(\alpha \in R)$ and $t_{\lambda}(\lambda \in L)$ the reflection with respect to $\alpha$ and the translation by $\lambda$, respectively. (Both are elements of $\widetilde{W}$.) It is known that $\left(W_{a}, S_{a}\right)$ is a Coxeter system for $S_{a}=S \cup\left\{s_{0}\right\}$. Put $N=$ the stabilizer of the set $S_{a}$ in $\widetilde{W}$. Then the splitting $\widetilde{W}=N \cdot W_{a}$ holds and the length function $l: W_{a} \rightarrow \mathbb{Z}_{\geq 0}$ naturally extends to that on $\widetilde{W}$ (the Iwahori-Matsumoto formula).

For $q=q_{s} \in K^{\times}$, we define the Hecke algebra of $\widetilde{W}, \widetilde{H}=H(\widetilde{W}, q)$, as the $K$-algebra with basis $T_{w}(w \in \widetilde{W})$ satisfying the following relations:

$$
\begin{gathered}
\left(T_{s}+1\right)\left(T_{s}-q\right)=0 \quad\left(s \in S_{a}\right), \\
T_{w} \cdot T_{w^{\prime}}=T_{w w^{\prime}} \quad \text { if } l(w)+l\left(w^{\prime}\right)=l\left(w w^{\prime}\right) .
\end{gathered}
$$

This $\widetilde{H}$ contains the Hecke algebra $H=H(W, q)$ of $\S 1$ as a subalgebra. For simplicity we put $T_{\lambda}=T_{t_{\lambda}}$. We call $\lambda \in L$ dominant if $\left\langle\lambda, a^{\vee}\right\rangle \geq 0$ for all $\alpha \in R^{+}$, where $\langle$,$\rangle is the canonical pairing on L \times L^{\vee}$. Following Bernstein, we put $\widetilde{T}_{\lambda}=T_{\lambda+\mu} T_{\mu}^{-1} \quad(\lambda \in L)$, where $\mu$ is chosen such that both $\mu$ and $\lambda+\mu$ are dominant. Then it is known that $\widetilde{T}_{\lambda}$ is well defined and the set consisting of the element $\widetilde{T}_{w}=T_{x} \widetilde{T}_{\lambda} \quad\left(w=x t_{\lambda}\right)$ for all $w \in W$ is a basis of $\widetilde{H}$. Put $\widetilde{H}_{\phi}=\sum_{\lambda \in L} K \cdot \widetilde{T}_{\lambda}$. This is a commutative subalgebra of $\widetilde{H}$ naturally isomorphic to the group algebra $K[L]$. We have $\widetilde{H}=H \cdot \widetilde{H}_{\phi} \simeq H \otimes_{K} \widetilde{H}_{\phi}$. For $I \subset S$, put $\widetilde{H}_{I}=H_{I} \cdot \widetilde{H}_{\phi}$. It is known that $\widetilde{H}_{I}$ is a subalgebra of $\widetilde{H}$, which is isomorphic to a Hecke algebra of $\widetilde{W}_{I}=W_{I} \ltimes L$. (Here we use the root data $\left(L, R_{I}, L^{\vee}, R_{I}^{\vee}\right)$ of $M_{I}$, the standard Levi subgroup of type $I$ of $G$; note that the length function for $\widetilde{W}_{I}$ is not equal to the restriction of that for $\widetilde{W}$ to $\widetilde{W}_{I}$.)

Now we define the induced module $\operatorname{Ind}_{I} N$ for an $\widetilde{H}_{I}$-module $N$ by $\operatorname{Ind}_{I} N=$ $\widetilde{H} \otimes_{\widetilde{H}_{I}} N$. The restriction of an $\widetilde{H}$-module $M$ to $\widetilde{H}_{I}$ is denoted as before by $\operatorname{Res}_{I} M$. Let $\widetilde{R}$ be the Grothendieck group of finite-dimensional $\widetilde{H}$-modules over $K$. For $[M](M$ is an $\widetilde{H}$-module), we define its dual by the same formula as in $\S 1$ :

$$
D[M]=\sum_{I \subset S}(-1)^{|I|}\left[\operatorname{Ind}_{I}\left(\operatorname{Res}_{I} M\right)\right]
$$


Put $T_{w}^{*}=(-1)^{l(x)} q^{l(w)} T_{w^{-1}}^{-1}$ for $w=x t_{\lambda} \in \widetilde{W}(x \in W, \lambda \in L)$. This * defines an involutive automorphism of $\widetilde{H}$, coincides with the previous ${ }^{*}$ in $\S 1$ on $H$, and defines a twisted $\widetilde{H}$-module $M^{*}$ for each $\widetilde{H}$-module $M$.

Theorem 2. We have $D[M]=\left[M^{*}\right]$ for any $\widetilde{H}$-module $M$.

The proof of Theorem 2 is similar to the proof of Theorem 1 in $\S 1$. We use $\widetilde{H} \otimes_{\widetilde{H}_{\phi}} M$ instead of $H \otimes_{K} M:$ The canonical decomposition $\widetilde{H}=H \otimes_{K} \widetilde{H}_{\phi}$ gives an isomorphism $\widetilde{H} \otimes_{\widetilde{H}_{\phi}} M \simeq H \otimes_{K} M$. By using this, we define $\tau_{s} \in$ $\operatorname{End}_{K}\left(\widetilde{H} \otimes_{\widetilde{H}_{\phi}} M\right)$ for $s \in S$ as in (1.1) and have

$$
\operatorname{Ind}_{I}\left(\operatorname{Res}_{I} M\right) \simeq \widetilde{H} \otimes_{\widetilde{H}_{\phi}} M / \sum_{s \in S} L_{s} \quad\left(L_{s}=\operatorname{Im} \tau_{s}\right) .
$$

(Note here that $L_{s}$ is an $\widetilde{H}$-submodule of $\widetilde{H} \otimes_{\widetilde{H}_{\phi}} M$.) Hence we get, by the same argument as in $\S 1$,

$$
D[M]=[(\mathrm{id} \otimes \pi)(\chi)(1 \otimes M)],
$$

where $\chi=\sum_{w \in W}(-1)^{l(w)} T_{w} \otimes \pi\left(T_{w}\right)^{-1} \in \widetilde{H} \otimes_{\widetilde{H}_{\phi}} \widetilde{H}$. Note that the tensor product is taken over $\widetilde{H}_{\phi}$. Therefore we have only to show the next two lemmas.

Lemma 2. $\left(T_{s_{0}} \otimes 1\right) \chi=\chi\left(1 \otimes\left(-q T_{s_{0}}^{-1}\right)\right)$ in $\widetilde{H} \otimes_{\widetilde{H}_{\phi}} \tilde{H}$.

Lemma 3. $\left(T_{\gamma} \otimes 1\right) \chi=(-1)^{l(x)} \chi\left(1 \otimes T_{\gamma}\right)$ for $\gamma \in N$ with $\gamma=x t_{\lambda} \quad(x \in W, \lambda \in$ $L)$ in $\widetilde{H} \otimes_{\widetilde{H}_{\phi}} \widetilde{H}$.

Proof of Lemma 2. It is known that (see, e.g., [Kt, 1.9])

$$
T_{s_{0}} T_{y}= \begin{cases}\widetilde{T}_{s_{0} y} & \text { if } y^{-1}\left(\alpha_{0}\right)>0, \\ q \widetilde{T}_{s_{0} y}+(q-1) \widetilde{T}_{y} & \text { if } y^{-1}\left(\alpha_{0}\right)<0\end{cases}
$$

for $y \in W\left(\right.$ since $\left.T_{y}=\widetilde{T}_{y}\right)$. Hence we get

$$
\left(T_{s_{0}} \otimes 1\right)\left(T_{y} \otimes T_{y}^{-1}-T_{s_{\alpha_{0}} y} \otimes T_{s_{\alpha_{0}} y}^{-1}\right)=\left(T_{y} \otimes T_{y}^{-1}-T_{s_{\alpha_{0}} y} \otimes T_{s_{\alpha_{0}} y}^{-1}\right)\left(1 \otimes\left(-q T_{s_{0}}^{-1}\right)\right)
$$

for $y \in W$ with $y^{-1}\left(\alpha_{0}\right)>0$, noting that

$$
T_{y} \otimes T_{y}^{-1}=\widetilde{T}_{w} \otimes \widetilde{T}_{w}^{-1} \quad\left(w=y t_{\lambda} ; y \in W, \lambda \in L\right)
$$

in $\widetilde{H} \otimes_{\widetilde{H}_{\phi}} \widetilde{H}$. This implies Lemma 2 .

Proof of Lemma 3. Since $\gamma=x t_{\lambda}$ satisfies $l(\gamma)=0$, we have

$$
T_{\gamma} \widetilde{T}_{y t_{\mu}}=T_{\gamma} T_{y t_{\mu}}=T_{x y t_{y-1}(\lambda)+\mu}=\widetilde{T}_{x y t_{y-1}(\lambda)+\mu}
$$

for $\mu$ sufficiently dominant (i.e., $\left\langle\lambda, \alpha^{\vee}\right\rangle \gg 0$ for all $\alpha>0$ ). Hence we get

$$
\left(T_{\gamma} \otimes 1\right)\left(T_{y} \otimes T_{y}^{-1}\right)=\left(T_{x y} \otimes T_{x y}^{-1}\right)\left(1 \otimes T_{\gamma}\right)
$$

in $\widetilde{H} \otimes_{\widetilde{H}_{\phi}} \widetilde{H}$. This proves Lemma 3 and thus completes the proof of Theorem 2. 
Remark. Let $k$ be a nonarchimedean local field, and $\mathscr{G}$ the group of $k$-rational points of a connected split reductive group over $k$ whose dual group is given by $G$. We denote by $\mathscr{P}_{I}$ the standard parabolic subgroup of $\mathscr{G}$ corresponding to $I \subset S$, and by $\mathscr{U}_{I}$ the unipotent radical of $\mathscr{P}_{I}$. Then we define the duality operator on $\mathscr{R}(\mathscr{G})$, the Grothendieck group of finitely generated admissible $\mathscr{G}$-modules over $\mathbb{C}$, by

$$
D[V]=\sum_{I \subset S}(-1)^{|I|}\left[\operatorname{Ind}_{\mathscr{P}_{I}}^{\mathscr{G}}\left(V_{\mathscr{U}_{I}}\right)\right]
$$

for a $\mathscr{G}$-module $V$. Here $V_{\mathscr{U}_{I}}$ is the Jacquet module associated to $\mathscr{P}_{I}$. By [B] and [M], we see that the duality operator for $\widetilde{H}$-modules in Theorem 2 is equivalent to this duality operator for admissible $\mathscr{G}$-modules with nonzero Iwahori subgroup fixed vectors. In particular, for such a $\mathscr{G}$-module $V$, we see that $D^{2}[V]=[V]$ and $D[V]$ is represented by an irreducible module if $V$ is irreducible. It may be conjectured that a similar result $\left(D^{2}=\right.$ id and, for an irreducible $V$, there exists an irreducible $V^{\prime}$ so that $\left.\pm D[V]=\left[V^{\prime}\right]\right)$ holds for general $\mathscr{G}$-modules (cf. [Z]). Exactly the analogous result is known to be true for finite Chevalley groups (see [A, Kw, DL]).

\section{ACKNOWLEDGMENT}

The author expresses his gratitude to the referee for pointing out an error contained in the first version of this paper.

\section{REFERENCES}

[A] D. Alvis, The duality operation in the character ring of a finite Chevalley groups, Bull. Amer. Math. Soc. (N.S.) 1 (1979), 907-911.

[B] A. Borel, Admissible representations of a semi-simple group over a local field with vectors fixed under an Iwahori subgroup, Invent. Math. 35 (1976), 233-259.

[C] C. W. Curtis, Truncation and duality in the character ring of a finite group of Lie type, J. Algebra 62 (1980), 320-332.

[DL] P. Deligne and G. Lusztig, Duality for representations of reductive groups over a finite field, I, II, J. Algebra 74 (1982), 284-291; ibid. 81 (1983), 540-545.

[Kt] S. Kato, On the Kazhdan-Lusztig polynomials for affine Weyl groups, Adv. in Math. 55 (1985), 103-130.

[Kw] N. Kawanaka, Fourier transforms of nilpotently supported invariant functions on a semisimple Lie algebra over a finite field, Invent. Math. 69 (1982), 411-435.

[M] H. Matsumoto, Analyse harmonique dans les systems de Tits bornologiques de type affine, Lecture Notes in Math, vol. 590, Springer-Verlag, Berlin, Heidelberg, and New York, 1979.

[SI] L. Solomon, The orders of the finite Chevalley groups, J. Algebra 3 (1966), 376-393.

[Sp] T. A. Springer, Reductive groups, Proc. Sympos. Pure Math., vol. 33, part 1, Amer. Math. Soc., Providence, RI, 1979, pp. 3-27.

[Z] A. V. Zelevinskii, p-adic analogue of the Kazhdan-Lusztig hypothesis, Functional Anal. Appl. 15 (1981), 83-92.

Division of Mathematics, Department of Fundamental Sciences, Faculty of InteGRATEd Human StUdies, Kyoto University, Kyoto 606-01, JAPAN

E-mail address: kato@platon.kula.kyoto-u.ac.jp 